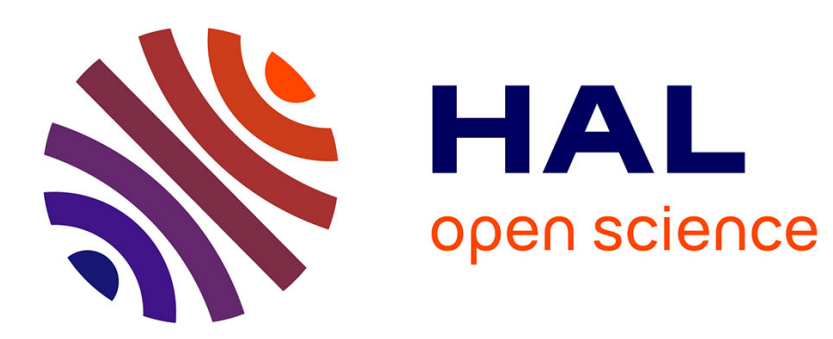

\title{
Existence of solutions for semi-linear equations involving the $p$-Laplacian: the non coercive case
}

Isabeau Birindelli, Françoise Demengel

\section{To cite this version:}

Isabeau Birindelli, Françoise Demengel. Existence of solutions for semi-linear equations involving the $p$-Laplacian: the non coercive case. Calculue of Variations and partial differential equations, 2004, 20 (4), pp.343-366. hal-00842589

\section{HAL Id: hal-00842589 \\ https://hal.science/hal-00842589}

Submitted on 8 Jul 2013

HAL is a multi-disciplinary open access archive for the deposit and dissemination of scientific research documents, whether they are published or not. The documents may come from teaching and research institutions in France or abroad, or from public or private research centers.
L'archive ouverte pluridisciplinaire HAL, est destinée au dépôt et à la diffusion de documents scientifiques de niveau recherche, publiés ou non, émanant des établissements d'enseignement et de recherche français ou étrangers, des laboratoires publics ou privés. 


\title{
Existence of solutions for semi-linear equations involving the $p$-Laplacian : the non coercive case
}

\author{
Isabeau Birindelli \\ Università di Roma "La Sapienza" \\ Françoise Demengel \\ University of Cergy-Pontoise
}

\section{Introduction}

In this paper we give necessary and sufficient conditions for the existence of solutions of the following equation

$$
\left\{\begin{array}{lc}
-\operatorname{div}\left(|\nabla u|^{p-2} \nabla u\right)+(g-\lambda) u^{p-1}=f u^{q-1}, & u \geq 0 \text { in } \Omega \\
u=0 & \text { on } \partial \Omega,
\end{array}\right.
$$

where $\Omega$ is a bounded smooth domain of $\mathbb{R}^{N}, 1<p<N, p<q \leq \frac{p N}{N-p}:=p^{\star}$, $f$ and $g$ belong to $L^{\infty}$, and $\lambda \in \mathbb{R}$. By solution of (1.1), we mean a function $u \in W_{0}^{1, p}(\Omega)$ satisfying (1.1) in the weak usual sense.

In particular we shall study (1.1) considering the position of $\lambda$ with respect to the principal eigenvalue. Precisely, it is well known that the concept of "eigenvalue" and "eigenfunction" has been generalized by many authors to the quasi-linear setting of the $p$-Laplacian $\Delta_{p}:=\operatorname{div}\left(|\nabla \cdot|^{p-2} \nabla.\right)$, in particular let us recall the works of Allegretto and Huang in [2], Anane in [3] and Lindqvist in [19]. We shall now state their definitions and the principal properties obtained in the works cited above. 
Definition $1.1 \lambda_{1}$ the first "eigenvalue" of $-\operatorname{div}\left(|\nabla \cdot|^{p-2} \nabla.\right)+g$ in $W_{0}^{1, p}(\Omega)$ is defined by

$$
\lambda_{1}:=\inf _{\left\{\psi \in W_{0}^{1, p}(\Omega),|\psi|_{p}=1\right\}}\left\{\int_{\Omega}|\nabla \psi|^{p}+\int_{\Omega} g|\psi|^{p}\right\} .
$$

It is by now a classical result that there exists $\phi$, positive in $\Omega$ for which this infimum is achieved. $\phi$ is called the "eigenfunction" corresponding to $\lambda_{1}$.

In particular $\phi$ satisfies

$$
\left\{\begin{array}{lc}
-\operatorname{div}\left(|\nabla \phi|^{p-2} \nabla \phi\right)+\left(g-\lambda_{1}\right) \phi^{p-1}=0 & \text { in } \Omega \\
\phi=0 & \text { on } \partial \Omega .
\end{array}\right.
$$

Furthermore $\phi$ is simple, i.e. any solution of (1.2) satisfies $v=k \phi$ for some $k \in \mathbb{R}$. In the sequel we will normalize $\phi$ in the $L^{p}(\Omega)$ norm.

Clearly for any $\lambda<\lambda_{1}$ the only nonnegative solution of

$$
\left\{\begin{array}{lc}
-\operatorname{div}\left(|\nabla u|^{p-2} \nabla u\right)+(g-\lambda) u^{p-1}=0 & \text { in } \Omega \\
u=0 & \text { on } \partial \Omega
\end{array}\right.
$$

is $u \equiv 0$.

On the other hand $\lambda_{1}$ is isolated, i.e. there exists $\delta>0$ such that for any $\lambda$ in $\left(\lambda_{1}, \lambda_{1}+\delta\right)$ the only solution of (1.3) is $u \equiv 0$ as well.

Our first results concern some necessary conditions for the existence of solutions.

Theorem 1.2 Suppose that there exists a nonnegative solution $u \not \equiv 0$ of equation (1.1). Then

1) For $\lambda<\lambda_{1}$, the set $\Omega^{+}$defined as

$$
\Omega^{+}:=\{x \in \Omega, f(x)>0\}
$$

is nonempty.

2) For $\lambda>\lambda_{1}, \Omega^{-}:=\{x \in \Omega, f(x)<0\} \neq \emptyset$ and $\int_{\Omega} f \phi^{q}<0$.

3) For $\lambda=\lambda_{1}, \Omega^{+} \neq \emptyset, \Omega^{-} \neq \emptyset$ and $\int_{\Omega} f \phi^{q}<0$.

Theorem 1.3 There exists $\lambda^{\prime}>\lambda_{1}$ such that there are no non trivial non negative solutions of equation (1.1) for $\lambda>\lambda^{\prime}$. 
Theorem 1.4 Suppose that there exists $\bar{\lambda}>\lambda_{1}$ for which (1.1) possesses a solution. Then, (1.1) has a solution for $\left.\lambda \in] \lambda_{1}, \bar{\lambda}\right]$.

Our next result concerns the existence of solutions of equation (1.1) in the sub-critical case:

Theorem 1.5 Suppose that $\Omega^{+}$and $\Omega^{-}$are nonempty, that $p<q<p^{\star}$, and $\int_{\Omega} f \phi^{q}<0$. Then there exists $\delta>0$ such that for $\lambda \in\left(\lambda_{1}, \lambda_{1}+\delta\right)$ equation (1.1) has at least two non zero and nonnegative solutions of equation (1.1). For $\lambda=\lambda_{1}$ there exists at least one solution of (1.1) nonnegative and not identically zero.

Remark 1: The solutions are obtained as minima of the two variational problems:

$$
\alpha_{\lambda, q}=\inf _{\left\{u \in W_{o}^{1, p}(\Omega), \int_{\Omega} f|u|^{q}=-1\right\}}\left\{\int_{\Omega}|\nabla u|^{p}+\int_{\Omega}(g-\lambda)|u|^{p}\right\}
$$

and

$$
\mu_{\lambda, q}=\inf _{\left\{u \in W_{o}^{1, p}(\Omega), \int_{\Omega} f|u|^{q}=1\right\}}\left\{\int_{\Omega}|\nabla u|^{p}+\int_{\Omega}(g-\lambda)|u|^{p}\right\} .
$$

Indeed, if $u \in W_{o}^{1, p}(\Omega)$ realizes $\alpha_{\lambda, q}$ (respectively $\mu_{\lambda, q}$ ), so does $|u|$, and it is easy to see that $u$ satisfies:

$$
-\operatorname{div}\left(|\nabla u|^{p-2} \nabla u\right)+(g-\lambda) u^{p-1}=-\alpha_{\lambda, q} f u^{q-1}
$$

(respectively

$$
\left.-\operatorname{div}\left(|\nabla u|^{p-2} \nabla u\right)+(g-\lambda) u^{p-1}=\mu_{\lambda, q} f u^{q-1}\right) .
$$

By a standard scaling argument one obtains two nonnegative solutions of equation (1.1), one being such that $\int_{\Omega} f u^{q}>0$ and the other such that $\int_{\Omega} f u^{q}<0$.

For simplicity of notation let $\alpha_{\lambda}:=\alpha_{\lambda, p^{\star}}$ and $\mu_{\lambda}:=\mu_{\lambda, p^{\star}}$.

Theorem 1.6 Suppose that $q=p^{\star}$ and that $\Omega^{+}, \Omega^{-} \neq \emptyset$, that $\lambda>\lambda_{1}$ and that $\int_{\Omega} f \phi^{p^{*}}<0$. Then there exists $\delta>0$ such that if $\lambda \in\left(\lambda_{1}, \lambda_{1}+\delta\right)$ there exists at least one solution of equation (1.1). If moreover,

$$
\mu_{\lambda}<K(N, p)^{-p} \sup |f|^{\frac{-p}{p^{\star}}},
$$

then, there exist at least two non zero solutions of equation (1.1). 
Remark 2: As in the subcritical case, the solutions are obtained as minima of $\alpha_{\lambda}$ and $\mu_{\lambda}$.

Remark 3: According to Theorems 1.4 and 1.5 the solutions of equation (1.1) exist for an interval, $\left(\lambda_{1}, \bar{\lambda}\right)$. On the other hand for some $\left.\lambda \in\right] \lambda_{1}, \bar{\lambda}[$, there may be only one solution, because for $\lambda$ not close to $\lambda_{1}$ nothing can be said about the sign of $\int_{\Omega} f u_{\lambda}^{q}$ when $u_{\lambda}$ is a solution obtained by Theorem 1.4.

For $p=2$ i.e. the classical Laplacian and $2<q<\frac{2 n}{n-2}$ problem (1.1) has been extensively studied when $f>0$. Since we are concerned with the case where $f$ changes sign, let us recall the main results in that case. Necessary and sufficient conditions for the existence of solutions for (1.1) have been given by Alama and Tarantello [1], Berestycki, Capuzzo Dolcetta and Nirenberg [5] and Ouyang [20] in the non coercive case.

Alama and Tarantello in [1] and the authors of the present paper in [6] have studied the critical case i.e. $q=\frac{2 n}{n-2}$. Let us also mention the very interesting work of Chen and $\mathrm{Li}$ in [7].

It is well known that the $p$-Laplacian appears in many contexts : NonNewtonian fluids, nonlinear elasticity and reaction diffusion problems just to name a few. Indeed equation (1.1) has been extensively studied for general $p$ and $q$; in particular for $q$ critical, existence of solutions of problem (1.1) was studied by Guedda and Veron in [14] for $f \equiv 1, g(x) \equiv \lambda=0$. Demengel and Hebey in [10] gave existence of variational solutions when $f$ changes sign and the functional $\int_{\Omega}|\nabla u|^{p}+\int_{\Omega}(g-\lambda)|u|^{p}$ is coercive i.e. $\lambda<\lambda_{1}$.

In [12], the authors study a similar problem with $(g-\lambda) u^{p-1}$ replaced by cteu $^{k-1}$ with $k \neq p$.

Always for general $p$ but $q$ subcritical the non coercive case was also studied by Drabek and Pohozaev in [11]; they use the fibering method to obtain some existence results for $\lambda$ close to $\lambda_{1}$. See also Pohozaev and Veron [21] for the Neumann problem.

Finally for $q$ critical, Drabek and Huang studied the problem in $\mathbb{R}^{N}[10]$, while Arioli and Gazzola in [4] proved existence for solutions changing sign through a linking method.

The above Theorems are the natural extension to the $p$-Laplacian of the results obtained in [6]. Nonetheless the proofs differ from the case $p=2$. In particular the proofs of Theorems 1.5 and 1.6 follow the approach taken by Ouyang in [20]. Although we should mention that Ouyang treats the 
sub-critical case and he uses bifurcation technic that don't hold for $p \neq 2$.

The outline of the paper is the following. In the next section we prove the necessary conditions (i.e. Theorem 1.2 and 1.3) using among other things Picone's identity for the p-Laplacian (cf Allegretto and Huang [2]). In the third section we prove the existence results first for the sub-critical case and then for the critical case. Finally in the last section we construct some test functions to show that the condition on $\mu_{\lambda}$ of Theorem 1.6 can be satisfied and easily verified.

\section{Proofs of Theorem 1.2, 1.3, 1.4.}

Let us recall Picone's identity for the $p$-Laplacian as formulated by Allegretto and Huang in [2]. Suppose that $v$ and $w$ belong to $W^{1, p}(\Omega)$ with $v \geq 0$ and $w>0$, then

$$
|\nabla v|^{p}-\nabla\left(\frac{v^{p}}{w^{p-1}}\right) \cdot \sigma(w) \geq 0
$$

everywhere in $\Omega$, for $\sigma(w):=|\nabla w|^{p-2} \nabla w$.

Moreover if equality holds then $w=k v$ for some constant $k \in \mathbb{R}$.

Proof of Theorem 1.2

Since in the case $\lambda<\lambda_{1}$ the functional

$$
I_{\lambda}(u):=\int_{\Omega}|\nabla u|^{p}+\int_{\Omega}(g-\lambda)|u|^{p}
$$

is coercive the first assertion is obvious.

Let us prove 2. Suppose that $\lambda>\lambda_{1}$, and let $u$ be a nonnegative solution of (1.1) . Adapting the strict maximum principle of Vasquez, one has $u>0$ inside $\Omega$. In addition, from regularity results of [13], [23], [17], [9], $u$ is $\mathcal{C}^{1, \alpha}(\bar{\Omega})$, for every $\alpha \in[0,1[$. Using once more the strict maximum principle inspired from Hopf's lemma, as given in [24], one has the existence of some real $\epsilon>0$ such that $\phi \geq \epsilon u$ on $\bar{\Omega}$. As a consequence, one is allowed to multiply the equation $(1.1)$ by $(u)^{1-q} \phi^{q}$. Integrating by parts on $\Omega$, one obtains

$$
\int_{\Omega} f \phi^{q}=\int_{\Omega} \sigma(u) \cdot \nabla\left(u^{1-q} \phi^{q}\right)+\int_{\Omega}(g-\lambda) u^{p-1} u^{1-q} \phi^{q}
$$




$$
\begin{aligned}
& =(1-q) \int_{\Omega}|\nabla u|^{p}\left(\frac{\phi}{u}\right)^{q}+q \int_{\Omega}(\sigma(u) \cdot \nabla \phi)\left(\frac{\phi}{u}\right)^{q-1} \\
& +\int_{\Omega}(g-\lambda) u^{p-q} \phi^{q} .
\end{aligned}
$$

Now we multiply equation (1.2) by $\phi^{q-p+1} u^{p-q}$ and integrate over $\Omega$;

$$
\int_{\Omega} \sigma(\phi) \cdot \nabla\left(\phi^{q-p+1} u^{p-q}\right)+\int_{\Omega}\left(g-\lambda_{1}\right) \phi^{q} u^{p-q}=0
$$

and then

$$
\begin{aligned}
(q-p+1) \int_{\Omega}|\nabla \phi|^{p}\left(\frac{\phi}{u}\right)^{q-p} & +(p-q) \int_{\Omega} \sigma(\phi) \cdot \nabla u\left(\frac{\phi}{u}\right)^{q-p+1}+ \\
& +\int_{\Omega}\left(g-\lambda_{1}\right) \phi^{q} u^{p-q}=0 .
\end{aligned}
$$

Subtracting (2.4) to (2.5), one gets

$$
\begin{aligned}
(q-p+1) \int_{\Omega}|\nabla \phi|^{p}\left(\frac{\phi}{u}\right)^{q-p} & +(p-q) \int_{\Omega} \sigma(\phi) \cdot \nabla u\left(\frac{\phi}{u}\right)^{q-p+1} \\
-q \int_{\Omega}\left(\frac{\phi}{u}\right)^{q-1} \nabla \phi \cdot \sigma(u) & +(q-1) \int_{\Omega}\left(\frac{\phi}{u}\right)^{q}|\nabla u|^{p}+ \\
\left(\lambda-\lambda_{1}\right) \int_{\Omega} \phi^{q} u^{p-q} & =-\int_{\Omega} f \phi^{q} .
\end{aligned}
$$

Now apply Picone's identity as follows

$$
|\nabla u|^{p}-\nabla\left(\frac{u^{p}}{\phi^{p-1}}\right) \cdot \sigma(\phi) \geq 0 .
$$

Multiplying it by $\left(\frac{\phi}{u}\right)^{q}$ and integrating over $\Omega$ it becomes

$$
\begin{aligned}
\int_{\Omega}|\nabla u|^{p}\left(\frac{\phi}{u}\right)^{q} & -p \int_{\Omega} \nabla u \cdot \sigma(\phi) u^{p-q-1} \phi^{q-p+1}+ \\
& +(p-1) \int_{\Omega}|\nabla \phi|^{p} u^{p-q} \phi^{q-p} \geq 0 .
\end{aligned}
$$


Similarly, exchanging the role of $u$ and $\phi$ i.e. considering

$$
|\nabla \phi|^{p}-\nabla\left(\frac{\phi^{p}}{u^{p-1}}\right) \cdot \sigma(u) \geq 0
$$

and multiplying by $\left(\frac{\phi}{u}\right)^{q-p}$ one gets

$$
\begin{aligned}
\int_{\Omega}|\nabla \phi|^{p}\left(\frac{\phi}{u}\right)^{q-p} & -p \int_{\Omega}\left(\frac{\phi}{u}\right)^{q-1} \nabla \phi \cdot \sigma(u) \\
& +(p-1) \int_{\Omega}\left(\frac{\phi}{u}\right)^{q}|\nabla u|^{p} \geq 0 .
\end{aligned}
$$

Multiply (2.8) by $\frac{q}{p}$ and (2.7) by $\frac{q}{p}-1$ their sum gives

$$
\begin{gathered}
(q-p+1) \int_{\Omega}|\nabla \phi|^{p}\left(\frac{\phi}{u}\right)^{q-p}+(p-q) \int_{\Omega} \nabla u \cdot \sigma(\phi)\left(\frac{\phi}{u}\right)^{q-p+1}+ \\
-q \int_{\Omega}\left(\frac{\phi}{u}\right)^{q-1} \nabla \phi \cdot \sigma(u)+(q-1) \int_{\Omega}|\nabla u|^{p}\left(\frac{\phi}{u}\right)^{q} \geq 0
\end{gathered}
$$

Substracting (2.9) from (2.6) we obtain

$$
\int_{\Omega} f \phi^{q}+\left(\lambda-\lambda_{1}\right) \int_{\Omega} \phi^{q} u^{p-q} \leq 0 .
$$

When $\lambda>\lambda_{1}$, this implies that $\int_{\Omega} f \phi^{q}<0$ and 2) is proved.

For the proof of 3 ), let $\lambda=\lambda_{1}$ and let $u$ be a nonnegative solution of equation (1.1). Multiplying it by $u$ one obtains

$$
\int_{\Omega}|\nabla u|^{p}+\int_{\Omega}\left(g-\lambda_{1}\right) u^{p}=\int_{\Omega} f u^{q} .
$$

Since the functional $I_{\lambda_{1}}$ is non negative, one has $\int_{\Omega} f u^{q} \geq 0$. Suppose that it is zero. Then $u$ would be an eigenfunction for the eigenvalue $\lambda_{1}$, which would imply that $f u^{q-1}=0$. Then $u$ must be zero on a set of positive measure, which contradicts the fact that $u$ is parallel to $\phi>0$ in $\Omega$. We have proved that $\int_{\Omega} f u^{q}>0$, this implies that $\Omega^{+} \neq \emptyset$.

We shall now prove that $\int_{\Omega} f \phi^{q}<0$, this of course implies also that $\Omega^{-} \neq \emptyset$. 
From the previous computations in the proof of 2), and precisely from (2.6) with $\lambda=\lambda_{1}$ and from (2.9), we obtain that

$$
\begin{aligned}
(q-p+1) \int_{\Omega}|\nabla \phi|^{p}\left(\frac{\phi}{u}\right)^{q-p} & +(p-q) \int_{\Omega} \nabla u \cdot \sigma(\phi)\left(\frac{\phi}{u}\right)^{q-p+1} \\
-q \int_{\Omega}\left(\frac{\phi}{u}\right)^{q-1} \nabla \phi \cdot \sigma(u) & +(q-1) \int_{\Omega}|\nabla u|^{p}\left(\frac{\phi}{u}\right)^{q}+ \\
& =-\int_{\Omega} f \phi^{q} .
\end{aligned}
$$

As a consequence $\int_{\Omega} f \phi^{q} \leq 0$. Suppose by contradiction that $\int_{\Omega} f \phi^{q}=0$, then the left hand side of the previous identity is zero. Recalling (2.8) and (2.9)the left hand side is a sum of two nonnegative quantities, hence they must be both null. Therefore we have obtained that

$$
\begin{aligned}
\int_{\Omega}|\nabla \phi|^{p}\left(\frac{\phi}{u}\right)^{q-p} & -p \int_{\Omega}\left(\frac{\phi}{u}\right)^{q-1} \nabla \phi \cdot \sigma(u) \\
& +(p-1) \int_{\Omega}\left(\frac{\phi}{u}\right)^{q}|\nabla u|^{p}=0
\end{aligned}
$$

and

$$
\begin{aligned}
\int_{\Omega}|\nabla u|^{p}\left(\frac{\phi}{u}\right)^{q} & -p \quad \int_{\Omega} \nabla u \cdot \sigma(\phi) u^{p-q-1} \phi^{q-p+1}+ \\
+ & (p-1) \int_{\Omega}|\nabla \phi|^{p} u^{p-q} \phi^{q-p}=0 .
\end{aligned}
$$

Clearly (2.12) and (2.13) imply that

$$
|\nabla u|^{p}-\nabla\left(\frac{u^{p}}{\phi^{p-1}}\right) \cdot \sigma(\phi)=0
$$

and

$$
|\nabla \phi|^{p}-\nabla\left(\frac{\phi^{p}}{u^{p-1}}\right) \cdot \sigma(u)=0 .
$$

Each of these identities implies that $\phi$ is parallel to $u$. Then $u$ is an eigenfunction. This implies that $f u^{q-1}$ is identically zero which is a contradiction. 
Proof of Theorem 1.3.

Let $B$ be a ball on which $f>0, B \subset \subset \Omega^{+}$. Let then $\left(\psi, \mu^{\star}\right)$ be the non zero and non negative normalized solution, of

$$
\begin{cases}-\Delta_{p} \psi+\left(-\mu^{\star}\right) \psi^{p-1}=0 & \text { in } B \\ \psi=0 & \text { on } \partial B\end{cases}
$$

Suppose that a solution of equation (1.1) exists for $\lambda$ such that $|g|_{\infty}+\mu^{\star}<$ $\lambda, u \geq 0$ and non identically zero. On $B$, by the strict maximum principle of Vasquez, $u>0$. Using Picone's identity, one has

$$
|\nabla \psi|^{p}-\nabla\left(\frac{\psi^{p}}{u^{p-1}}\right) \cdot \sigma(u) \geq 0
$$

in $B$, hence, integrating over $B$

$$
0 \leq \int_{B}\left(\mu^{\star}\right) \psi^{p}+\int_{B}(g-\lambda) \psi^{p}
$$

here, we have used the fact that $\psi=0$ on $\partial B$ and the equation verified by $u$, since

$$
-\Delta_{p} u+(g-\lambda) u^{p-1}=f u^{q-1} \geq 0
$$

on $B$. (2.14) of course contradicts the choice of $\lambda$.

Proof of Theorem 1.4.

Let $\bar{\lambda}$ be such that $\lambda_{1}<\bar{\lambda}$ and take $\left.\lambda \in\right] \lambda_{1}, \bar{\lambda}[$. Let $\bar{u}$ be a solution of (1.1) for $\bar{\lambda}$. Then $\bar{u}$ is a supersolution of (1.1) for $\lambda$. Indeed

$$
-\Delta_{p} \bar{u}+(g-\lambda) \bar{u}^{p-1}=f \bar{u}^{q-1}+(\bar{\lambda}-\lambda) \bar{u}^{p-1} \geq f \bar{u}^{q-1}
$$

and $\bar{u}=0$ on the boundary. On another hand, taking $\epsilon$ small enough, $\epsilon \phi$ is a subsolution, since

$$
-\Delta_{p}(\epsilon \phi)+(g-\lambda)(\epsilon \phi)^{p-1}=\left(\lambda_{1}-\lambda\right) \epsilon^{p-1} \phi^{p-1} \leq f \epsilon^{q-1} \phi^{q-1},
$$

(using $p<q$ and $\left.\left(\lambda_{1}-\lambda\right) \epsilon^{p-1} \phi^{p-1}<0\right)$. Moreover, using strong maximum principle of Vasquez and regularity results, one can choose $\epsilon$ small enough in order to have $\bar{u} \geq \epsilon \phi$. Finally we use the following Proposition, whose proof can be found in the appendix and is a mere adaptation of the classical sub and super solution for $p=2$. (see e.g. [15], see also [22]): 
Proposition 2.1 Suppose that $f(x, t)=a(x)|t|^{q-2} t+b(x)|t|^{p-2} t$ with $1<$ $p<q$ with $a$ and $b$ two continuous and bounded functions on $\Omega$ Suppose that $\bar{u}$ is a weak supersolution for $-\Delta_{p} u+f(x, u), \bar{u}=0$ on $\partial \Omega$, and that $\underline{u}$ is a weak subsolution with $\underline{u}=0$ on $\partial \Omega$. Suppose that there exists some constant $c$ and $C$ such that

$$
-\infty<c \leq \underline{u} \leq \bar{u} \leq C<+\infty
$$

Then, there exists a solution $u$ between $\underline{u}$ and $\bar{u}$

Using this Proposition with $f(x, u)=(g-\lambda) u^{p-1}-f u^{q-1}$, and $\underline{u}=\epsilon \phi$, one obtains that there exists a solution which is such that

$$
\epsilon \phi \leq u \leq \bar{u}
$$

\section{$3 \quad$ Existence of solutions}

Proof of Theorem 1.5

This proof is inspired by the arguments used in [20]. We begin with the subcritical case. Suppose that $q<p^{\star}$. Let us recall the following notations:

$$
\begin{gathered}
\lambda_{q}^{\star}=\inf _{\left\{u \in W_{0}^{1, p}(\Omega),|u|_{p}^{p}=1, \int_{\Omega} f u^{q}=0\right\}}\left\{\int_{\Omega}|\nabla u|^{p}+\int_{\Omega}\left(g-\lambda_{1}\right)|u|^{p}\right\} \\
\alpha_{\lambda, q}=\inf _{\left\{u, \int_{\Omega} f|u|^{q}=-1\right\}}\left\{\int_{\Omega}|\nabla u|^{p}+\int_{\Omega}(g-\lambda)|u|^{p}\right\}
\end{gathered}
$$

and

$$
\mu_{\lambda, q}=\inf _{\left\{u, \int_{\Omega} f|u|^{q}=1\right\}}\left\{\int_{\Omega}|\nabla u|^{p}+\int_{\Omega}(g-\lambda)|u|^{p}\right\} .
$$

Let $I_{\lambda}(u):=\int_{\Omega}|\nabla u|^{p}+\int_{\Omega}(g-\lambda)|u|^{p}$.

We will prove the following facts

1. $\lambda_{q}^{\star}>0$.

2. For $\lambda \in] \lambda_{1}, \lambda_{1}+\lambda_{q}^{\star}\left[, \alpha_{\lambda, q}<0\right.$ and it is achieved; $\alpha_{\lambda_{1}, q}=0$.

3. For $\lambda \in] \lambda_{1}, \lambda_{1}+\lambda_{q}^{\star}\left[, \mu_{\lambda, q}>0\right.$ and it is achieved. Moreover $\mu_{\lambda_{1}, q}>0$. 


\section{Proof of 1.}

By the definition of $\lambda_{1}, \lambda_{q}^{\star} \geq 0$. Suppose by contradiction that $\lambda_{q}^{\star}=0$. Let $\left(u_{n}\right)$ be a minimizing sequence. Since $|\nabla| u_{n}||=\left|\nabla u_{n}\right|$, one can assume that $u_{n} \geq 0$. Since $\left|u_{n}\right|_{p}=1$ and $\int_{\Omega}\left|\nabla u_{n}\right|^{p}+\int_{\Omega}\left(g-\lambda_{1}\right) u_{n}^{p} \rightarrow 0$, then $\int_{\Omega}\left|\nabla u_{n}\right|^{p}$ is bounded; hence $\left(u_{n}\right)$ is bounded in $W_{0}^{1, p}$. Extracting from it a subsequence and passing to the limit, one gets that there exists some $u \geq 0$, weak limit of $\left(u_{n}\right)$ in $W^{1, p}(\Omega)$, such that

$$
\int_{\Omega}|\nabla u|^{p}+\int_{\Omega}\left(g-\lambda_{1}\right) u^{p} \leq 0
$$

Clearly (3.17) implies that

$$
\int_{\Omega}|\nabla u|^{p}+\int_{\Omega}\left(g-\lambda_{1}\right) u^{p}=0
$$

and then $u$ is an eigenfunction for $\lambda_{1}$ and then it is parallel to $\phi$. Moreover $u \in W_{0}^{1, p}, \int_{\Omega}|u|^{p}=1$ and $\int_{\Omega} f u^{q}=0$, which contradicts the assumption $\int_{\Omega} f \phi^{q}<0$. Finally $\lambda_{q}^{\star}>0$.

\section{Proof of 2.}

In order to prove that $\alpha_{\lambda, q}<0$ for $\lambda>\lambda_{1}$, let us take, as an admissible function, $v=\frac{\phi}{\left(-\int_{\Omega} f \phi^{q}\right)^{\frac{1}{q}}}$. We then have

$$
\alpha_{\lambda, q} \leq I_{\lambda}(v)=\frac{1}{\left(-\int_{\Omega} f \phi^{q}\right)^{\frac{p}{q}}} I_{\lambda}(\phi)=\frac{1}{\left(-\int_{\Omega} f \phi^{q}\right)^{\frac{p}{q}}}\left(\lambda_{1}-\lambda\right)<0 .
$$

Now we will check that

$$
\alpha_{\lambda, q}>-\infty
$$

If not, there would exist a subsequence $\left(u_{i}\right), u_{i} \geq 0$ for all $i$, such that $\int_{\Omega} f u_{i}^{q}=-1$ and $I_{\lambda}\left(u_{i}\right) \rightarrow-\infty$. Clearly $\left|u_{i}\right|_{p} \rightarrow+\infty$ since

$$
\varlimsup \int_{\Omega}(g-\lambda) u_{i}^{p} \leq \alpha_{\lambda, q}
$$

Let $w_{i}=\frac{u_{i}}{\left|u_{i}\right|_{p}}$. One has $\int_{\Omega} f w_{i}^{q} \rightarrow 0$, and $\left(w_{i}\right)$ is bounded in $W_{0}^{1, p}(\Omega)$, since $\left|w_{i}\right|_{p}=1$ and $\int_{\Omega}\left|\nabla w_{i}\right|^{p}+\int_{\Omega}(g-\lambda) w_{i}^{p}=\frac{I_{\lambda}\left(u_{i}\right)}{\left|u_{i}\right|_{p}^{p}} \leq 0$ implies

$$
\int_{\Omega}\left|\nabla w_{i}\right|^{p} \leq|g-\lambda|_{\infty}
$$


Then, there exists a subsequence still denoted $\left(w_{i}\right)$, such that $w_{i} \rightarrow w$ weakly in $W^{1, p}(\Omega)$. Observe that

$$
\int_{\Omega}|w|^{p}=1 \text { and } I_{\lambda}(w) \leq 0 .
$$

This contradicts the definition of $\lambda$, since $\int_{\Omega} f w^{q}=0$ and $\left.\lambda \in\right] \lambda_{1}, \lambda_{1}+\lambda_{q}^{\star}[$. We have proved that $\alpha_{\lambda, q}>-\infty$.

We shall now see that $\alpha_{\lambda, q}$ is achieved. Let $\left(u_{n}\right), u_{n} \geq 0$ be a minimizing sequence for $\alpha_{\lambda, q}$ i.e.

$$
\begin{gathered}
\int_{\Omega}\left|\nabla u_{n}\right|^{p}+\int_{\Omega}(g-\lambda) u_{n}^{p} \rightarrow \alpha_{\lambda, q}, \\
\int_{\Omega} f u_{n}^{q}=-1 .
\end{gathered}
$$

Let us prove first that $\left|u_{n}\right|_{p}$ is bounded. If not, one can argue as previously by considering $w_{n}=\frac{u_{n}}{\left|u_{n}\right|_{p}}$. It is easy to see that $\left(w_{n}\right)$ converges weakly in $W^{1, p}(\Omega)$, up to a subsequence, towards some function $w \geq 0$ which satisfies $\int_{\Omega} f w^{q}=0,|w|_{p}=1$ and

$$
\int_{\Omega}|\nabla w|^{p}+\int_{\Omega}(g-\lambda) w^{p}=0 .
$$

This contradicts the definition of $\lambda$. Hence $\int_{\Omega}\left|u_{n}\right|^{p}$ is bounded, and so is $\int_{\Omega}\left|\nabla u_{n}\right|^{p}$. By extracting from $\left(u_{n}\right)$ a subsequence, one obtains that there exists $u \in W_{0}^{1, p}, u \geq 0$, such that $\int_{\Omega} f u^{q}=-1$ and by lower semi-continuity of the semi-norm $|\nabla u|_{p}$ with respect to the weak topology,

$$
\int_{\Omega}|\nabla u|^{p}+\int_{\Omega}(g-\lambda) u^{p} \leq \alpha_{\lambda, q} .
$$

Finally using the definition of $\alpha_{\lambda, q}, u$ is a minimizer for $\alpha_{\lambda, q}$, hence it is a nonzero solution of

$$
-\operatorname{div}\left(|\nabla u|^{p-2} \nabla u\right)+(g-\lambda) u^{p-1}=-\alpha_{\lambda, q} f u^{q-1} .
$$

Proof of 3.

Acting as we did for $\alpha_{\lambda, q}$ one can prove that $\mu_{\lambda, q}>-\infty$. We are now going to check that $\mu_{\lambda, q}$ is achieved. 
Indeed, let $u_{n}$ be a sequence such that $u_{n} \geq 0$,

$$
\begin{gathered}
\int_{\Omega}\left|\nabla u_{n}\right|^{p}+\int_{\Omega}(g-\lambda) u_{n}^{p} \rightarrow \mu_{\lambda, q}, \\
\int_{\Omega} f u_{n}^{q}=1 .
\end{gathered}
$$

Suppose that $\left|u_{n}\right|_{p} \rightarrow \infty$. Then considering $w_{n}=\frac{u_{n}}{\left|u_{n}\right|_{p}}$ one gets, by passing to the limit that there exists $w \geq 0$, a weak limit of $\left(w_{n}\right)$ in $W^{1, p}(\Omega)$, such that

$$
\int_{\Omega}|\nabla w|^{p}+\int_{\Omega}(g-\lambda) w^{p} \leq 0
$$

and $\int_{\Omega} f w^{q}=0$, which contradicts the assumption $\left.\lambda \in\right] \lambda_{1}, \lambda_{1}+\lambda_{q}^{\star}[$. Then $\left(u_{n}\right)$ is bounded and we pass to the limit to obtain

$$
\int_{\Omega}|\nabla u|^{p}+\int_{\Omega}(g-\lambda) u^{p}=\mu_{\lambda, q}
$$

and $\int_{\Omega} f u^{q}=1$. Hence $\mu_{\lambda, q}$ is achieved.

For $\lambda=\lambda_{1}, \mu_{\lambda_{1}, q} \geq 0$, but since it is achieved, if $\mu_{\lambda_{1}, q}=0$, we would have an eigenfunction $u$ such that $\int_{\Omega} f u^{q}=1$, which contradicts the assumptions. Then $\mu_{\lambda_{1}, q}>0$.

For $\lambda>\lambda_{1}$ let $u_{q} \geq 0$ which realizes the minimum in $\mu_{\lambda, q}$. Then :

$$
-\Delta_{p} u_{q}+(g-\lambda) u_{q}^{p-1}=\mu_{\lambda, q} f u_{q}^{q-1} .
$$

Using the procedure of the proof of Theorem 1.2 for $u_{q}$, inequality $(2.10)$ becomes

$$
\mu_{\lambda, q} \int_{\Omega} f \phi^{q}+\left(\lambda-\lambda_{1}\right) \int_{\Omega} \phi^{q} u_{q}^{p-q} \leq 0 .
$$

Using $\int_{\Omega} f \phi^{q}<0$ and $\lambda-\lambda_{1}>0$, one gets $\mu_{\lambda, q}>0$.

Let us now state and prove some results concerning $\alpha_{\lambda, q}$ and $\mu_{\lambda, q}$.

Lemma 3.1 The following convergences hold:

$$
\begin{gathered}
\lim _{\lambda \rightarrow \lambda_{1}} \alpha_{\lambda, q}=\alpha_{\lambda_{1}, q}=0, \\
\lim _{\lambda \rightarrow \lambda_{1}} \mu_{\lambda, q}=\mu_{\lambda_{1}, q}
\end{gathered}
$$


Lemma 3.2 1. $\lambda_{p^{\star}}^{\star} \geq \varlimsup_{q \rightarrow p^{\star}} \lambda_{q}^{\star} \geq \varliminf_{q \rightarrow p^{\star}} \lambda_{q}^{\star}:=\lambda^{\star}>0$.

2. For $\lambda_{1} \leq \lambda<\lambda_{1}+\lambda^{\star}$, then $0 \leq \varliminf_{q \rightarrow p^{\star}} \mu_{\lambda, q} \leq \varlimsup_{q \rightarrow p^{\star}} \mu_{\lambda, q} \leq \mu_{\lambda}(=$ $\left.\mu_{\lambda, p^{\star}}\right)$.

3. For $\lambda$ close to $\lambda_{1}, \alpha_{\lambda}\left(=\alpha_{\lambda, p^{\star}}\right)>-\infty$ and $\overline{\lim }_{q \rightarrow p^{\star}} \alpha_{\lambda, q} \leq \alpha_{\lambda}$.

Proof of Lemma 3.1

Suppose by contradiction that (3.18) does not hold, then there exist some number $\alpha<0$ and a sequence of $\lambda \in \mathbb{R}, \lambda \rightarrow \lambda_{1}$, and $\left(u_{\lambda}\right) \subset W_{o}^{1, p}(\Omega)$ such that

$$
\int_{\Omega}\left|\nabla u_{\lambda}\right|^{p}+\int_{\Omega}(g-\lambda)\left|u_{\lambda}\right|^{p} \leq \alpha .
$$

Moreover one can assume that $u_{\lambda} \geq 0$. If $\left(u_{\lambda}\right)$ is bounded, we may extract from it a subsequence weakly convergent to some $u \in W_{0}^{1, p}$, such that

$$
\int_{\Omega}|\nabla u|^{p}+\int_{\Omega}\left(g-\lambda_{1}\right) u^{p} \leq \alpha<0
$$

which is absurd.

On the other hand if $\left(u_{\lambda}\right)$ diverges we can normalize it and then we obtain a sequence $\left(w_{\lambda}\right)$ such that $\int_{\Omega}\left|w_{\lambda}\right|^{p}=1$. By extracting a subsequence, there exists $w \geq 0$, such that $\int_{\Omega}|w|^{p}=1, \int_{\Omega} f w^{q}=0$ and

$$
\int_{\Omega}|\nabla w|^{p}+\int_{\Omega}\left(g-\lambda_{1}\right) w^{p} \leq 0 .
$$

This would imply that $w$ is parallel to $\phi$ which is absurd since $\int_{\Omega} f \phi^{q}<0$.

Let us now prove (3.19). Let us define $\bar{\mu}_{q}:=\overline{\lim }_{\lambda \rightarrow \lambda_{1}} \mu_{\lambda, q}$. One already has $\bar{\mu}_{q} \leq \mu_{\lambda_{1}, q}$. Let $u_{\lambda}$ which satisfies $u_{\lambda} \geq 0$ and

$$
\begin{gathered}
-\Delta_{p} u_{\lambda}+(g-\lambda) u_{\lambda}^{p-1}=\mu_{\lambda, q} f u_{\lambda}^{q-1} \\
\int_{\Omega} f u_{\lambda}^{q}=1 .
\end{gathered}
$$

As we did above, one can prove that $\left(u_{\lambda}\right)$ is bounded in the $W^{1, p}$ norm. By extracting a subsequence, one gets by passing to the limit when $\lambda \rightarrow \lambda_{1}$

$$
\int_{\Omega}|\nabla u|^{p}+\int_{\Omega}\left(g-\lambda_{1}\right) u^{p} \leq \bar{\mu}_{q}
$$

and $u \geq 0, \int_{\Omega} f u^{q}=1$. This clearly implies that $\bar{\mu}_{q} \geq \mu_{\lambda_{1}, q}$ and gives the required result. 
Proof of Lemma 3.2

Let us prove 1, and first that $\underline{\lim }_{q \rightarrow p^{\star}} \lambda_{q}^{\star}>0$. Since $\lambda_{q}^{\star}$ is achieved, let $u_{q} \geq 0$ be a solution of

$$
\int_{\Omega}\left|\nabla u_{q}\right|^{p}+\int_{\Omega}\left(g-\lambda_{1}\right) u_{q}^{p}=\lambda_{q}^{\star}
$$

$\left|u_{q}\right|_{p}=1$ and $\int_{\Omega} f u^{q}=0$. Suppose by contradiction that $\underline{\lim }_{q \rightarrow p^{\star}} \lambda_{q}^{\star}=0$. Then, by extracting from $\left(u_{q}\right)$ a subsequence, one gets by passing to the limit when $q$ tends to $p^{\star}$ :

$$
\int_{\Omega}|\nabla u|^{p}+\int_{\Omega}\left(g-\lambda_{1}\right) u^{p} \leq 0
$$

and $|u|_{p}=1$. Since $I_{\lambda_{1}}$ is coercive, $\int_{\Omega}|\nabla u|^{p}+\int_{\Omega}\left(g-\lambda_{1}\right) u^{p}=0$, and the sequence $\int_{\Omega}\left|\nabla u_{q}\right|^{p}$ tends to $\int_{\Omega}|\nabla u|^{p}$. Hence $u_{q}$ tends to $u$ strongly in $W^{1, p}(\Omega)$, and finally $\int_{\Omega} f u^{p^{\star}}=\lim _{q \rightarrow p^{\star}} \int_{\Omega} f u_{q}^{q}=0$. This is a contradiction since $\phi$ is simple and $\int_{\Omega} f \phi^{p^{\star}}<0$. As a consequence $\lambda^{\star}>0$.

We now prove that $\lambda^{\star} \leq \lambda_{p^{\star}}^{\star}$. Indeed, let $u \geq 0$ be a $\mathcal{C}^{1}$ function, such that $\int_{\Omega} f u^{p^{\star}}=0,|u|_{p}=1$, and

$$
I_{\lambda_{1}}(u) \leq \lambda_{p^{\star}}^{\star}+\epsilon
$$

If there exists an infinite sequence $q \rightarrow p^{\star}$, such that $\int_{\Omega} f u^{q}=0$, one has the desired result. If not, there exists an infinite sequence $q \rightarrow p^{\star}$ such that either $\int_{\Omega} f u^{q}>0$ for all $q$, or $\int_{\Omega} f u^{q}<0$ for all $q$. Suppose that we are in the first case and define $\alpha(q)=\frac{\int_{\Omega} f u^{q}}{\int_{\Omega} f u^{q}-\int_{\Omega} f \phi^{q}}$. Then $\alpha(q) \in[0,1]$, and $\alpha(q) \rightarrow 0$ when $q \rightarrow p^{\star}$. Let us define

$$
v_{q}=\left(\alpha(q) \phi^{q}+(1-\alpha(q)) u^{q}\right)^{\frac{1}{q}} .
$$

By the regularity properties of $\phi$ and $u, v_{q}$ belongs to $W_{0}^{1, p}(\Omega), v_{q} \geq 0$ and $\int_{\Omega} f v_{q}^{q}=0$ by the choice of $\alpha(q)$. Moreover it is easy to check that $v_{q}$ tends to $u$ in $W^{1, p}(\Omega)$ strongly. As a consequence

$$
\lambda_{q}^{\star}(1+o(1)) \leq \lambda_{q}^{\star}\left(\int_{\Omega} v_{\alpha}^{p}\right) \leq \int_{\Omega}\left|\nabla v_{\alpha}\right|^{p}+\int_{\Omega}\left(g-\lambda_{1}\right) v_{\alpha}^{p} \leq \lambda_{p^{\star}}^{\star}+\epsilon+o(1)
$$


when $q \rightarrow p^{\star}$. This implies that $\lambda^{\star} \leq \lambda_{p^{\star}}^{\star}$. Suppose now that there exists a sequence $q \rightarrow p^{\star}$ such that $\int_{\Omega} f u^{q}<0$. Let $u_{0}$ be nonnegative in $\mathcal{C}^{1}(\bar{\Omega})$, such that $\int_{\Omega} f u_{0}^{q}>0$ and define

$$
v_{\alpha}=\left(\alpha(q) u_{0}^{q}+(1-\alpha(q)) u^{q}\right)^{\frac{1}{q}}
$$

where $\alpha(q)=\frac{\int_{\Omega} f u^{q}}{\int_{\Omega} f u^{q}-\int_{\Omega} f u_{0}^{q}}$. One concludes as in the case $\int_{\Omega} f u^{q}>0$.

To prove 2 ., let $\varepsilon>0$ be given and let $u$ be such that $u \geq 0, \int_{\Omega} f u^{p^{\star}}=1$ and

$$
I_{\lambda}(u) \leq \mu_{\lambda}+\varepsilon
$$

Then for $q$ close to $p^{\star}, \int_{\Omega} f u^{q}>\frac{1}{2}$ and taking $v_{q}=\frac{u}{\left(\int_{\Omega} f u^{q}\right)^{\frac{1}{q}}}$, one gets, for $q$ sufficiently close to $p^{*}$,

$$
\mu_{\lambda, q} \leq I_{\lambda}\left(v_{q}\right) \leq \mu_{\lambda}+2 \varepsilon
$$

We will prove 3 . by contradiction. Hence suppose that there exists a sequence $\lambda_{n} \rightarrow \lambda_{1}$ and a sequence $\left(u_{n}\right), u_{n} \geq 0$ such that $\int_{\Omega} f u_{n}^{p^{\star}}=-1$ and $I_{\lambda_{n}}\left(u_{n}\right) \leq-n$. Clearly $\left|u_{n}\right|_{p} \rightarrow+\infty$. Then defining $w_{n}=\frac{u_{n}}{\left|u_{n}\right|_{p}}$, and extracting a subsequence from it, one gets that there exists $w \geq 0$ such that

$$
I_{\lambda_{1}}(w) \leq 0
$$

This in fact implies that strong convergence holds and then $\int_{\Omega} f w^{p^{\star}}=0$, which contradicts $|w|_{p}=1$ and $\phi$ is simple.

Before giving the proof of Theorem 1.6 let us recall one of the key ingredients employed herein i.e. the famous concentration compactness principle of P.L. Lions[18]:

Lemma 3.3 Let $\Omega$ be some bounded open set in $\mathbb{R}^{n}$, and $\left(u_{k}\right)$ be some sequence in $W_{o}^{1, p}(\Omega)$, which is bounded in $W^{1, p}(\Omega)$. Then there exist a subsequence of $\left(u_{k}\right)$, still denoted $\left(u_{k}\right)$ for simplicity, two nonnegative measures $\mu$ 
and $\nu$ on $\bar{\Omega}$, a sequence of points $x_{i}$ in $\bar{\Omega}$, two sequences of nonnegative real numbers $\mu_{i}$ and $\nu_{i}$ and a function $u$ in $W_{o}^{1, p}(\Omega)$, such that

$$
\left|\nabla u_{k}\right|^{p} \rightarrow \mu \geq|\nabla u|^{p}+\sum_{i} \mu_{i} \delta_{x_{i}}
$$

(the convergence being tight on $\bar{\Omega}$ i.e. $\int_{\Omega}\left|\nabla u_{k}\right|^{p} \rightarrow \int_{\bar{\Omega}} \mu$,),

$$
\left|u_{k}\right|^{p^{\star}} \rightarrow \nu=|u|^{p^{\star}}+\sum_{i} \nu_{i} \delta_{x_{i}}
$$

(the convergence being tight on $\bar{\Omega}$ i.e. $\int_{\Omega}\left|u_{k}\right|^{p^{\star}} \rightarrow \int_{\bar{\Omega}} \nu$ ), with the inequality

$$
\nu_{i} \leq K(n, p)^{\frac{p^{\star}}{p}} \mu_{i}
$$

Proof of Theorem 1.6

First part.

We prove the existence of solutions for $\alpha_{\lambda}$ and for $\lambda$ sufficiently close to $\lambda_{1}$. According to Lemma 3.1 above, $\lim _{\lambda \rightarrow \lambda_{1}} \alpha_{\lambda}=0$. One takes $\lambda$ sufficiently close to $\lambda_{1}$ in order to have $-\alpha_{\lambda}<K(N, p)^{-p}(\sup |f|)^{\frac{-p}{p^{\star}}}$, and $\lambda<\lambda_{1}+\lambda^{\star}$. Let $\left(u_{q}\right), u_{q} \geq 0$ be a solution for the problem defining $\alpha_{\lambda, q}$.

Claim: $\left(u_{q}\right)_{q}$ is bounded in $L^{p}$.

Suppose that it is not true. Then, proceeding as in the proof of Theorem 1.5 , there would exist a sequence $\left(w_{q}\right)$ such that $w_{q} \geq 0,\left|w_{q}\right|_{p}=1$, and

$$
\int_{\Omega}\left|\nabla w_{q}\right|^{p}+\int_{\Omega}(g-\lambda) w_{q}^{p} \leq 0 .
$$

Extracting from $\left(w_{q}\right)$ a subsequence one obtains that there exists $w$, weak limit of $w_{q}$ in $W^{1, p}$ such that $w \geq 0,|w|_{p}=1$, and

$$
\int_{\Omega}|\nabla w|^{p}+\int_{\Omega}(g-\lambda) w^{p} \leq 0
$$

If $\int_{\Omega} f w^{p^{\star}}=0$, this contradicts the assumption $\lambda<\lambda_{1}+\lambda^{\star} \leq \lambda_{1}+\lambda_{p^{\star}}^{\star}$. If $\int_{\Omega} f w^{p^{\star}}>0, I_{\lambda}(w) \geq \mu_{\lambda}\left(\int_{\Omega} f w^{p^{\star}}\right)^{\frac{p}{p^{\star}}}>0$, and since $\mu_{\lambda} \geq 0$ one would obtain that $\mu_{\lambda}=0=I_{\lambda}(w)$, and using lower semi-continuity for the weak topology

$$
I_{\lambda}(w) \leq \underline{\lim }_{q \rightarrow p^{\star}} I_{\lambda}\left(w_{q}\right) \leq 0 .
$$


Finally $I_{\lambda}(w)=\lim _{q \rightarrow p^{\star}} I_{\lambda}\left(w_{q}\right)$ and then $\int_{\Omega}\left|\nabla w_{q}\right|^{p} \rightarrow \int_{\Omega}|\nabla w|^{p}$, strong convergence holds in fact, hence $\int_{\Omega} f w^{p^{*}}=\lim _{q \rightarrow p^{\star}} \int_{\Omega} f w_{q}^{q}=0$, which is a contradiction of the assumption $\int_{\Omega} f w^{p^{\star}}>0$.

Finally suppose that $\int_{\Omega} f w^{p^{\star}}<0$. Then, applying P.L. Lions' concentration compactness lemma recalled above, one gets that there exists two bounded and nonnegative measures $\mu$ and $\nu$ on $\bar{\Omega}$, some countable set of points $\left(x_{i}\right)$ in $\bar{\Omega}$, and some sequence of non-negative numbers $\left(\mu_{i}\right)$ and $\left(\nu_{i}\right)$, which satisfy, up to a subsequence

$$
\begin{gathered}
\left|\nabla w_{q}\right|_{p}^{p}-\mu \geq|\nabla w|_{p}^{p}+\sum_{i} \mu_{i} \delta_{x_{i}} \\
\left|w_{q}\right|^{q}-\nu=|w|^{p^{\star}}+\sum_{i} \nu_{i} \delta_{x_{i}} .
\end{gathered}
$$

Passing to the limit in (3.22), in the equality $\int_{\Omega} f w_{q}^{q}=\frac{-1}{\left|u_{q}\right|_{p}^{\mid}}$, and using (3.23) and (3.24), one obtains

$$
\begin{gathered}
I_{\lambda}(w) \leq-\sum_{i} \mu_{i}, \\
\int_{\Omega} f w^{p^{*}}+\sum_{i} \nu_{i} f\left(x_{i}\right)=0 .
\end{gathered}
$$

On the other hand, using $\int_{\Omega} f w^{p^{*}}<0$, one has

$$
\alpha_{\lambda}\left(-\int_{\Omega} f w^{p^{\star}}\right)^{\frac{p}{p^{\star}}} \leq I_{\lambda}(w) \leq-\sum_{i} \mu_{i} .
$$

Hence,

$$
\sum_{i} \mu_{i} \leq-\alpha_{\lambda}\left(\sum_{i} \nu_{i} f\left(x_{i}\right)\right)^{\frac{p}{p^{*}}}
$$

Finally

$$
\sum_{i} \mu_{i} \leq-\alpha_{\lambda} \sum_{i}\left(\nu_{i} f\left(x_{i}\right)\right)^{\frac{p}{p^{\star}}} \leq-\alpha_{\lambda} \sup |f|^{\frac{p}{p^{\star}}} K(N, p)^{p} \sum_{i} \mu_{i} \leq \delta \sum_{i} \mu_{i}
$$

for some $\delta<1$. One obtains that $\mu_{i}=0$ and then $\nu_{i}=0$, as well as $\int_{\Omega} f w^{p^{\star}}=0$, which contradicts the assumption.

As a consequence the claim is proved i.e. $\left(u_{q}\right)$ is bounded in $L^{p}$.

Furthermore, since

$$
\alpha_{\lambda, q} \geq\left(\lambda_{1}-\lambda\right) \int_{\Omega}\left|u_{q}\right|^{p}
$$


the sequence $\alpha_{\lambda, q}$ is bounded too. Let us denote by $\bar{\alpha}$ the limit of a subsequence. Clearly $\bar{\alpha} \leq \alpha_{\lambda}$. Since $\left(u_{q}\right),\left(u_{q} \geq 0\right)$ is bounded, one may extract a subsequence such that $u_{q} \rightarrow u$ in $W^{1, p}$. Let us recall that $u_{q}$ satisfies:

$$
\left\{\begin{array}{l}
-\Delta_{p} u_{q}+(g-\lambda) u_{q}^{p-1}=-\alpha_{\lambda, q} f u_{q}^{q-1} \\
\int_{\Omega} f u_{q}^{q}=-1
\end{array}\right.
$$

Let us denote by $\sigma$ the weak limit of a subsequence in $L^{\frac{p}{(p-1)}}(\Omega)$ of $\sigma_{q}:=$ $\left|\nabla u_{q}\right|^{p-1} \nabla u_{q}$. Then, passing to the limit in equation (3.25) one gets $u \geq 0$ and

$$
-\operatorname{div}(\sigma)+(g-\lambda) u^{p-1}=-\bar{\alpha} f u^{p^{\star}-1} .
$$

Using again P.L. Lions' concentration lemma, there exist two bounded and nonnegative measures $\mu$ and $\nu$ on $\bar{\Omega}$, some countable sets of points $\left(x_{i}\right)$ in $\bar{\Omega}$, and some sequence of nonnegative numbers $\left(\mu_{i}\right)$ and $\left(\nu_{i}\right)$, which satisfy, up to a subsequence

$$
\begin{gathered}
\left|\nabla u_{q}\right|_{p}^{p}-\mu \geq|\nabla u|_{p}^{p}+\sum_{i} \mu_{i} \delta_{x_{i}} \text { tightly on } \bar{\Omega}, \\
\left|u_{q}\right|^{q}-\nu=|u|^{p^{\star}}+\sum_{i} \nu_{i} \delta_{x_{i}}, \text { tightly on } \bar{\Omega} .
\end{gathered}
$$

Let us multiply equation (3.25) (resp. equation (3.26)) by $u_{q} \varphi$ (resp. $u \varphi$ ), for a function $\varphi$ in $\mathcal{D}(\bar{\Omega})$. One obtains

$$
\int_{\Omega}\left|\nabla u_{q}\right|^{p} \varphi+\int_{\Omega} \sigma_{q} \cdot \nabla \varphi u_{q}+\int_{\Omega}(g-\lambda) u_{q}^{p} \varphi=-\alpha_{\lambda, q} \int_{\Omega} f u_{q}^{q} \varphi
$$

and

$$
\int_{\Omega}(\sigma \cdot \nabla u) \varphi+\int_{\Omega}(\sigma \cdot \nabla \varphi) u+\int_{\Omega}(g-\lambda) u^{p} \varphi=-\bar{\alpha} \int_{\Omega} f u^{p^{\star}} \varphi .
$$

By passing to the limit in (3.27), one gets

$$
\int_{\Omega} \mu \varphi+\int_{\Omega}(\sigma \cdot \nabla \varphi) u+\int_{\Omega}(g-\lambda) u^{p} \varphi=-\bar{\alpha}\left(\int_{\Omega} f u^{p^{\star}} \varphi+\sum_{i} \nu_{i} f\left(x_{i}\right) \varphi\left(x_{i}\right)\right) .
$$

Subtracting (3.28) from (3.29) one obtains

$$
\int_{\Omega}(\mu-\sigma \cdot \nabla u) \varphi=-\bar{\alpha}\left(\sum_{i} \nu_{i} f\left(x_{i}\right) \varphi\left(x_{i}\right)\right)
$$


Using Lebesgue decomposition of $\mu:=\mu^{a c}+\mu^{s}$, where $\mu^{a c}$ is the absolutely continuous part of $\mu$, one derives

$$
\begin{gathered}
|\nabla u|^{p} \leq \mu^{a c}=\sigma \cdot \nabla u \\
\sum_{i} \mu_{i} \delta_{x_{i}} \leq \mu^{s}=-\bar{\alpha} \nu_{i} f\left(x_{i}\right) \delta_{x_{i}} .
\end{gathered}
$$

Suppose first that $x_{i}$ is such that $f\left(x_{i}\right) \leq 0$, then $\mu_{i}=\nu_{i}=0$.

On the other hand, passing to the limit in equation (3.27) and using lower semi-continuity one has

$$
I_{\lambda}(u) \leq \bar{\alpha}<0 .
$$

If $\int_{\Omega} f u^{p^{\star}}=0$ this contradicts the assumption $\left.\lambda \in\right] \lambda_{1}, \lambda_{1}+\lambda_{p^{\star}}^{\star}\left[\right.$. If $\int_{\Omega} f u^{p^{\star}}>0$ one also gets a contradiction, since

$$
0 \leq \mu_{\lambda}\left(\int_{\Omega} f u^{p^{\star}}\right)^{\frac{p}{p^{*}}} \leq I_{\lambda}(u)
$$

Suppose that $\int_{\Omega} f u^{p^{\star}}<0$, then using (3.31) and (3.28) one has

$$
\alpha_{\lambda}\left(-\int_{\Omega} f u^{p^{\star}}\right)^{\frac{p}{p^{\star}}} \leq I_{\lambda}(u) \leq-\bar{\alpha} \int_{\Omega} f u^{p^{\star}} \leq-\alpha_{\lambda} \int_{\Omega} f u^{p^{\star}} .
$$

From this, one obtains that $-\int_{\Omega} f u^{p^{\star}} \leq 1$.

On the other hand the identity

$$
\int_{\Omega} f u^{p^{\star}}+\sum_{i} \nu_{i} f\left(x_{i}\right)=-1
$$

yields to $\sum_{i} \nu_{i} f\left(x_{i}\right) \leq 0$, and since we are in the case $f\left(x_{i}\right) \geq 0$ we get $\nu_{i} f\left(x_{i}\right)=0$ for all $i$. Using (3.32) one obtains that $\int_{\Omega} f u^{p^{\star}}=-1$ and $\mu_{i}=0$. We have then

$$
\alpha_{\lambda} \leq \int_{\Omega}|\nabla u|^{p}+\int_{\Omega}(g-\lambda) u^{p} \leq \int_{\Omega} \sigma \cdot \nabla u+\int_{\Omega}(g-\lambda) u^{p}=\bar{\alpha} \leq \alpha_{\lambda}
$$

which implies that $\bar{\alpha}=\alpha_{\lambda}, \sigma . \nabla u=|\nabla u|^{p}$, the convergence of $\nabla u_{q}$ is strong in $W^{1, p}(\Omega)$ and $\alpha_{\lambda}$ is achieved. 
Second part. Since $\lim _{\lambda \rightarrow \lambda_{1}} \alpha_{\lambda}=\alpha_{\lambda_{1}}=0$, one can choose $\lambda$ sufficiently close to $\lambda_{1}$ in order to have

$$
\alpha_{\lambda}>-\left(\sup |f|^{\frac{p}{p^{\star}}} K(N, p)^{p}\right) .
$$

Now let $u_{q}$ be a function for which $\mu_{\lambda, q}$ is achieved, $u_{q} \geq 0$.

Claim: $\left(u_{q}\right)$ is bounded in $L^{p}$ when $q$ goes to $p^{\star}$.

Suppose on the contrary that $\left|u_{q}\right|_{p}$ tends to infinity. Then, defining $w_{q}=$ $\frac{u_{q}}{\left|u_{q}\right|_{p}}$, one obtains that $w_{q}$ tends, up to a subsequence, to a function $w \in$ $W_{0}^{1, p}(\Omega), w \geq 0$ which satisfies $|w|_{p}=1$, and

$$
\begin{gathered}
\int_{\Omega}|\nabla w|^{p}+\sum_{i} \mu_{i}+\int_{\Omega}(g-\lambda) w^{p} \leq 0, \\
\int_{\Omega} f w^{p^{\star}}+\sum_{i} \nu_{i} f\left(x_{i}\right)=0
\end{gathered}
$$

where $\left(\mu_{i}\right)$ and $\left(\nu_{i}\right)$ are as in the first part.

Suppose first that $\int_{\Omega} f w^{p^{\star}}=0$. Then one gets a contradiction with the conditions on $\lambda$ since

$$
\int_{\Omega}|\nabla w|^{p}+\int_{\Omega}(g-\lambda) w^{p} \leq 0
$$

Suppose that $\int_{\Omega} f w^{p^{\star}}>0$. Then by the definition of $\mu_{\lambda}$ one would obtain that

$$
\mu_{\lambda}\left(\int_{\Omega} f w^{p^{\star}}\right)^{\frac{p}{p^{\star}}} \leq|\nabla w|^{p}+\int_{\Omega}(g-\lambda) w^{p} \leq 0
$$

Since $\mu_{\lambda} \geq 0$, this may happen only if $\mu_{\lambda}=0$, and in the same time $I_{\lambda}(w)=$ 0 . Then, coming back to the previous inequalities, one has

$$
I_{\lambda}(w)=0 \leq \underline{\lim }_{q \rightarrow p^{\star}} I_{\lambda}\left(w_{q}\right) \leq 0
$$

hence $I_{\lambda}\left(w_{q}\right) \rightarrow I_{\lambda}(w)$, and strong convergence holds. This implies that $\int_{\Omega} f w^{p^{\star}}=\lim _{q \rightarrow p^{\star}} \int_{\Omega} f w_{q}^{q}=0$, which contradicts the assumption $\int_{\Omega} f w^{p^{\star}}>$ 0 .

Suppose finally that $\int_{\Omega} f w^{p^{\star}}<0$, then one can write

$$
\alpha_{\lambda}\left(-\int_{\Omega} f w^{p^{\star}}\right)^{\frac{p}{p^{\star}}} \leq \int_{\Omega}|\nabla w|_{p}^{p}+\int_{\Omega}(g-\lambda) w^{p} \leq-\sum_{i} \mu_{i}
$$


and then

$$
\begin{aligned}
\sum_{i} \mu_{i} & \leq\left(-\alpha_{\lambda}\right)\left(\sum_{i} \nu_{i}\left|f\left(x_{i}\right)\right|\right)^{\frac{p}{p^{\star}}} \\
& \leq\left(-\alpha_{\lambda}\right)\left(\sum_{i} \nu_{i}^{\frac{p}{p^{\star}}}\left|f\left(x_{i}\right)\right|^{\frac{p}{p^{\star}}}\right) \\
& \leq\left(-\alpha_{\lambda}\right) \sup |f|^{\frac{p}{p^{\star}}} \sum_{i} \mu_{i} K(N, p)^{p} \\
& \leq \delta \sum_{i} \mu_{i}
\end{aligned}
$$

for some $\delta<1$. Finally one has $\mu_{i}=0$ for all $i$ and then $\nu_{i}=0$. Then $\int_{\Omega} f w^{p^{\star}}=0$ which is absurd, as we remarked before. We have obtained that $\left(u_{q}\right)$ is bounded. This proves the claim.

Let $\beta=\frac{1}{2}\left(K(N, p)^{-p} \sup |f|^{\frac{-p}{p^{\star}}}-\mu_{\lambda_{1}}\right)$ and suppose that $\lambda$ is sufficiently close to $\lambda_{1}$ in order to ensure that

$$
\left|\alpha_{\lambda}\right|<\beta
$$

Let $\left(u_{q}\right)$ be a sequence of nonnegative minimizers for $\mu_{\lambda, q}, u_{q} \geq 0$. Then

$$
\begin{gathered}
-\Delta_{p} u_{q}+(g-\lambda) u_{q}^{p-1}=\mu_{\lambda, q} f u_{q}^{q-1} \\
\int_{\Omega} f u_{q}^{q}=1 .
\end{gathered}
$$

By the previous computations, the sequence $\left(u_{q}\right)$ is bounded in $L^{p}$, and since $\left(\mu_{\lambda, q}\right)$ is bounded too, $\left(u_{q}\right)$ is in fact bounded in $W^{1, p}$. Let us extract from it a subsequence such that

$$
u_{q} \rightarrow u
$$

in $W^{1, p}$ weakly. Let us denote by $\gamma$ the limit of some subsequence of $\mu_{\lambda, q}$. One has $\gamma \leq \mu_{\lambda} \leq \mu_{\lambda_{1}}$.

Acting as we did in the first part, one gets

$$
-\operatorname{div}(\sigma)+(g-\lambda) u^{p-1}=\gamma f u^{p^{\star}-1},
$$

denoting by $\sigma$ a weak limit of $\left|\nabla u_{q}\right|^{p-1} \nabla u_{q}$ in $L^{\frac{p}{p-1}}(\Omega)$. 
Multiplying equation (3.33) (respectively (3.34)) by $u_{q} \varphi$ (respectively by $u \varphi)$ with $\varphi \in \mathcal{D}(\bar{\Omega})$ and integrating over $\Omega$, introducing measures $\mu$ and $\nu$ as in the concentration compactness lemma one gets

$$
\begin{gathered}
\mu^{a c}-\sigma \cdot \nabla u=0 \\
\sum_{i} \mu_{i} \delta_{i} \leq \mu^{s}=\gamma \sum_{i} \nu_{i} f\left(x_{i}\right) \delta_{i} .
\end{gathered}
$$

This last identity yields that $\gamma$ cannot be zero: if it was, one would have $\mu_{i}=0$, hence $\nu_{i}=0$, and in the same time,

$$
\int_{\Omega}|\nabla u|^{p}+\int_{\Omega}(g-\lambda) u^{p}=0
$$

and

$$
\int_{\Omega} f u^{p^{\star}}=1
$$

This is impossible, since for example, one has supposed that $\lambda$ is not an eigenvalue. Then $\gamma>0$. Moreover, if $x_{i}$ is such that $f\left(x_{i}\right)<0$, then $\mu_{i}=0$, and so is $\nu_{i}$. Since one has

$$
|\nabla u|^{p} \leq \mu^{a c}=\sigma \cdot \nabla u
$$

coming back to (3.34), one gets

$$
\int_{\Omega}|\nabla u|^{p}+\int_{\Omega}(g-\lambda) u^{p} \leq \int_{\Omega} \sigma \cdot \nabla u+\int_{\Omega}(g-\lambda) u^{p}=\gamma \int_{\Omega} f u^{p^{\star}} .
$$

On another hand the identity

$$
\int_{\Omega} f u^{p^{\star}}+\sum_{i} \nu_{i} f\left(x_{i}\right)=1
$$

implies that $\sum_{i} \nu_{i} f\left(x_{i}\right) \leq 1$ if $\int_{\Omega} f u^{p^{\star}} \geq 0$. Suppose now that $\int_{\Omega} f u^{p^{\star}}<0$. Then $\nu_{f}=\sum_{i} \nu_{i} f\left(x_{i}\right)>1$. In the same time one has

$$
\alpha_{\lambda}\left(-\int_{\Omega} f u^{p^{\star}}\right)^{\frac{p}{p^{\star}}} \leq \int_{\Omega}|\nabla u|^{p}+\int(g-\lambda) u^{p} \leq \gamma \int_{\Omega} f u^{p^{\star}}
$$

and then

$$
\nu_{f} \leq 1+\left(\frac{-\alpha}{\gamma}\right)^{\frac{1}{1-\frac{p}{p^{\star}}}}
$$


As seen before if $f\left(x_{i}\right)<0, \mu_{i}=0$, hence $\nu_{i}=0$. If $f\left(x_{i}\right) \geq 0$, the previous calculations imply that for all $i, \nu_{i} f\left(x_{i}\right) \leq 1+\left(\frac{-\alpha}{\gamma}\right)^{\frac{1}{1-\frac{p}{p^{\star}}}}$. Finally

$$
\begin{aligned}
\mu_{i} & \leq \gamma\left(\frac{\nu_{i} f\left(x_{i}\right)}{1+\left(\frac{-\alpha}{\gamma}\right)^{\frac{1}{1-\frac{p}{p^{\star}}}}}\right)\left(1+\left(\frac{-\alpha}{\gamma}\right)^{\frac{1}{1-\frac{p}{p^{\star}}}}\right) \\
& \leq \gamma\left(\frac{\nu_{i} f\left(x_{i}\right)}{1+\left(\frac{-\alpha}{\gamma}\right)^{\frac{1}{1-\frac{p}{p^{\star}}}}}\right)^{1-\frac{p}{p^{\star}}}\left(\frac{\nu_{i} f\left(x_{i}\right)}{1+\left(\frac{-\alpha}{\gamma}\right)^{\frac{1}{1-\frac{p}{p^{\star}}}}}\right)\left(1+\left(\frac{-\alpha}{\gamma}\right)^{\frac{p}{p^{\star}}}\left(\frac{1-\frac{p}{p^{\star}}}{\gamma}\right)^{\frac{1}{1-\frac{p}{p^{\star}}}}\right)^{1-\frac{p}{p^{\star}}} K(N, p)^{p} \sup |f|^{\frac{p}{p^{\star}}} \mu_{i} \\
& \leq \gamma\left(1+\left(\frac{-\alpha}{\gamma}\right) K(N, p)^{p} \sup |f|^{\frac{p}{p^{\star}}} \mu_{i}\right. \\
& \leq \gamma\left(1+\frac{p}{x^{\frac{p}{p^{\star}}} \mu_{i}(\gamma-\alpha)}\right. \\
& \leq K(N, p)^{p} \sup \mid f \mu_{i}
\end{aligned}
$$

for some $\delta<1$. As a consequence $\mu_{i}=0$ and then $\nu_{i}=0$. Finally

$$
\begin{gathered}
\int_{\Omega} f u^{p^{\star}}=1 \\
\mu_{\lambda} \leq \int_{\Omega}|\nabla u|^{p}+\int_{\Omega}(g-\lambda)|u|^{p} \leq \int_{\Omega} \sigma . \nabla u+\int_{\Omega}(g-\lambda)|u|^{p} \leq \gamma
\end{gathered}
$$

hence $\mu_{\lambda}=\gamma,|\nabla u|^{p}=\sigma . \nabla u=\mu$, the convergence is strong, and $u$ is a minimizer for $\mu_{\lambda}$.

Remark 3.4 We have also obtained that $\mu_{\lambda}>0$.

Corollary 3.5 Suppose that $\int_{\Omega} f \phi^{p^{\star}}<0$ and that there exists a minimizer for $\lambda=\lambda_{1}$, then there exist at least two minimizers for $\lambda>\lambda_{1}$, and $\lambda$ sufficiently close to $\lambda_{1}$. 
Proof

Suppose that there exists a minimizer $u_{1}$ for the problem with $\lambda=\lambda_{1}$. Then

$$
\begin{aligned}
\inf _{\left\{u \in W_{0}^{1, p}(\Omega), \int_{\Omega} f|u|^{p^{\star}}=1\right\}}\left\{\int_{\Omega}|\nabla u|_{p}^{p}+\int_{\Omega}(g-\lambda) u^{p}\right\} & \leq I_{\lambda}\left(u_{1}\right)<I_{\lambda_{1}}\left(u_{1}\right) \\
& =\inf I_{\lambda_{1}}(u) \\
& \leq \frac{1}{K(N, p)^{p} \sup f(x)^{\frac{p}{p^{\star}}} .}
\end{aligned}
$$

As a consequence, using Theorem 1.6 one obtains that $I_{\lambda}$ has a minimizer.

\section{$4 \quad$ Estimates and test functions}

Let $x_{0} \in \mathbf{R}^{N}$ and $r=\left|x-x_{0}\right|$ the euclidean distance from $x_{0}$ to $x$. For $p>1$ given, $p$ real such that $p<N$, we define the function $u_{\epsilon}$ by

$$
u_{\epsilon}(x)=\left(\epsilon+r^{p / p-1}\right)^{1-N / p}
$$

and the function $v_{\epsilon}$ by

$$
v_{\epsilon}(x)=\left(\epsilon+r^{p / p-1}\right)^{1-N / p} \phi(r)
$$

where $\phi: \mathbf{R} \rightarrow \mathbf{R}$, nonnegative and smooth, is such that $\phi(r)=1$ for $r \leq \delta / 4$ and $\phi(r)=0$ for $r \geq \delta, \delta>0$ small. Recall here that

$$
u_{1}(x)=\left(1+r^{p / p-1}\right)^{1-N / p}
$$

realizes the best constant for the embedding of $W^{1, p}\left(\mathbf{R}^{N}\right)$ in $L^{p^{\star}}\left(\mathbf{R}^{N}\right)$. Let also $a$ and $f$ be smooth functions defined in a neighborhood $\Omega$ of $x_{0}$. We assume in what follows that $f>0$ in $B_{x_{0}}(\delta)$, and that $B_{x_{0}}(\delta) \subset \Omega$. For $u \in W_{0}^{1, p}(\Omega)$, we set

$$
I(u)=\frac{\int_{\Omega}|\nabla u|^{p} d x+\int_{\Omega}\left(g(x)-\lambda_{1}\right)|u|^{p} d x}{\left(\int_{\Omega} f(x)|u|^{p^{\star}} d x\right)^{\frac{p}{p^{\star}}}} .
$$

We also introduce

$$
k_{g}=0 \text { if } g\left(x_{0}\right)<\lambda_{1}
$$




$$
\begin{gathered}
k_{g}=\inf \left\{j \in \mathbf{N}, / j \geq 1 \text { and } \Delta^{j} g\left(x_{0}\right)<0\right\} \text { if not } \\
k_{f}=\inf \left\{j \in \mathbf{N}^{\star}, / \Delta^{j} f\left(x_{0}\right)<0\right\}
\end{gathered}
$$

with the convention that $k_{g}=+\infty$ (resp. $\left.k_{f}=+\infty\right)$ if the corresponding set above is empty. Here $\Delta^{j}=\Delta^{j-1} \circ \Delta, j \geq 1$, where $\Delta$ is the usual Laplacian. When $N>p^{2}$, we define as in [10], [6]

$$
k=\sup \left\{m \in \mathbf{N} / N>p^{2}+2 m(p-1)\right\}
$$

and for $j$ integer, we set

$$
\alpha_{N, j}=\frac{\Gamma\left(j+\frac{1}{2}\right) \Gamma\left(\frac{1}{2}\right)^{N-1}(2 j+N)}{\Gamma\left(j+\frac{N}{2}+1\right)}
$$

and

$$
\begin{gathered}
\tilde{\alpha}_{j}^{p, N}=\frac{\alpha_{N, j}}{(2 j) !} \int_{0}^{\infty} \frac{r^{N+2 j-1} d r}{\left(1+r^{\frac{p}{(p-1)}}\right)^{N-p}} \\
\tilde{\beta}_{j}^{p, N}=\frac{\alpha_{N, j}}{(2 j) !} \frac{(N-p)^{p}}{(p-1)^{p-1}} \int_{0}^{\infty} \frac{r^{N+2 j-1} d r}{\left(1+r^{\frac{p}{(p-1)}}\right)^{N}} .
\end{gathered}
$$

Note that $\tilde{\alpha}_{j}^{p, N}$ exists as soon as $N>p^{2}+2 j(p-1)$, that $\tilde{\beta}_{j}^{p, N}$ exists as soon as $N>2 j(p-1)$. One can find the explicit values of $\tilde{\alpha}_{j}^{p, N}, \tilde{\beta}_{j}^{p, N}$ in [10], Lemma 7.

Proposition 4.1 Suppose that $1<p^{2}<N$ and that $f$ and $g$ are $\mathcal{C}^{\infty}(\bar{\Omega})$. For $\epsilon>0$ sufficiently small,

$$
I\left(v_{\epsilon}\right)<\frac{1}{K(N, p)^{p} f\left(x_{0}\right)^{\frac{p}{p^{\star}}}}
$$

in each of the following cases

- 1. $k \geq k_{g}, k_{f}>k_{g}+\frac{p}{2}$, and $\Delta^{k_{g}}\left(g\left(x_{0}\right)-\lambda_{1}\right)<0$.

- 2. $k \geq k_{g}, k_{f}<k_{g}+\frac{p}{2}$, and $\Delta^{k_{f}} f\left(x_{0}\right)>0$.

- 3. $k \geq k_{g}, k_{f}=k_{g}+\frac{p}{2}$, and $\tilde{\alpha}_{k_{g}}^{p, n}\left(\Delta^{k_{g}}\left(g\left(x_{0}\right)-\lambda_{1}\right) f\left(x_{0}\right)-\tilde{\beta}_{k_{f}}^{p, n} \Delta^{k_{f}} f\left(x_{0}\right)<\right.$ 0 
- 4. $k \leq k_{g}, k_{f} \leq k+\frac{p}{2}$, and $\Delta^{k_{f}} f\left(x_{0}\right)>0$.

For example, the following corollary presents particular situations which enclose the results in the case where $p=2$ obtained in [6], see also [1] in the case $p=2$ and $g=0$ :

Corollary 4.2 Suppose that $1<p^{2}<n$. For $\epsilon>0$ small, one has that

$$
I\left(v_{\epsilon}\right)<\frac{1}{K(N, p)^{p} f\left(x_{0}\right)^{1-\frac{p}{N}}}
$$

in each of the following situations

- 1. $1<p<2$ and $g\left(x_{0}\right)<\lambda_{1}$.

- 2. $p=2$ and $\frac{8(N-1)}{(N-2)(N-4)}\left(g\left(x_{0}\right)-\lambda_{1}\right) f\left(x_{0}\right)-\Delta f\left(x_{0}\right)<0$.

- 3. $p>2$ and $g\left(x_{0}\right)=\lambda_{1}, \Delta g\left(x_{0}\right)=\Delta f\left(x_{0}\right)=0$ and $\Delta^{2} f\left(x_{0}\right)>0$.

As a consequence of Proposition 4.1 One obtains that if $f$ achieves its supremum on an interior point $x_{0}$ such that one of the situations described in 1. 2. 3. 4. occurs, then, there exists a solution to equation 1.1 for $\lambda=\lambda_{1}$ and for $\lambda$ close to $\lambda_{1}$.

We do not give the proofs of Proposition 4.1 and Corollary 4.2 , because they are very technical and are already written in [10], in the coercive case. One must just replace in [10] the function $a$ by the function $g-\lambda_{1}$.

\section{Appendix}

As mentioned in the introduction, in this appendix we want to prove the following

Proposition 2.1 Suppose that $f(x, t)=a(x)|t|^{q-2} t+b(x)|t|^{p-2} t$ with $1<$ $p<q$, and $a$ and $b$ two continuous and bounded functions on $\Omega$. Suppose that $\bar{u}$ is a weak supersolution for $-\Delta_{p} u+f(x, u) \bar{u}=0$ on $\partial \Omega$, and that $\underline{u}$ is a weak subsolution with $\underline{u}=0$ on $\partial \Omega$. Suppose that there exists some constant $c$ and $C$ such that

$$
-\infty<c \leq \underline{u} \leq \bar{u} \leq C<+\infty
$$


Then, there exists a solution $u$ between $\underline{u}$ and $\bar{u}$

Proof We follow the method of E. Hebey in [?].

Let $k$ be choosen in order that the function

$$
H(x, t)=f(x, t)+k|t|^{p-2} t
$$

be increasing on $\left[\inf _{x \in \bar{\Omega}} \underline{u}, \sup _{x \in \bar{\Omega}} \bar{u}\right]$. Let $u_{1}$ be the solution of the variational problem

$$
\inf _{u \in W_{0}^{1, p}(\Omega)} \frac{1}{p} \int_{\Omega}|\nabla u|^{p}+\frac{k}{p} \int_{\Omega}|u|^{p}-\int_{\Omega} H(x, \bar{u}) u .
$$

The solution $u_{1}$ is unique and satisfies the following partial differential equation

$$
-\Delta_{p} u_{1}+k\left|u_{1}\right|^{p-2} u_{1}=H(x, \bar{u})
$$

and in particular

$$
-\Delta_{p} u_{1}+k\left|u_{1}\right|^{p-2} u_{1} \leq-\Delta_{p} \bar{u}+k|\bar{u}|^{p-2} \bar{u}
$$

and by the comparison principle one gets that $u_{1} \leq \bar{u}$. On the other hand by the monotonicity properties of $H$

$$
-\Delta_{p} u_{1}+k\left|u_{1}\right|^{p-2} u_{1}=H(x, \bar{u}) \geq H\left(x, \underline{u} \geq-\Delta_{p} \underline{u}+k|\underline{u}|^{p-2} \underline{u}\right.
$$

and then

$$
u_{1} \geq \underline{u} .
$$

Finally $u_{1}$ is a supersolution since

$$
-\Delta_{p} u_{1}+k\left|u_{1}\right|^{p-2} u_{1}=H(x, \bar{u}) \geq H\left(x, u_{1}\right),
$$

hence

$$
\underline{u} \leq u_{1} \leq \bar{u}
$$

Iterating this process, one obtains the existence of a decreasing sequence $u_{n}$ of supersolutions and

$$
\underline{u} \leq u_{n} \leq \bar{u},
$$

with

$$
-\Delta_{p} u_{n}+k\left|u_{n}\right|^{p-2} u_{n}=H\left(x, u_{n-1}\right) .
$$


The sequence is, then, simply convergent and furthermore $u_{n}$ is bounded in $W^{1, p}$ since it is bounded in $L^{\infty}$ and

$\int_{\Omega}\left|\nabla u_{n}\right|^{p}+k \int_{\Omega}\left|u_{n}\right|^{p}-\int_{\Omega} H\left(x, u_{n-1}\right) u_{n} \leq \int_{\Omega}|\nabla \bar{u}|^{p}+k \int_{\Omega}|\bar{u}|^{p}-\int_{\Omega} H(x, \bar{u}) u$.

Extracting from it a subsequence one gets that there exists $u$ such that $u_{n} \rightarrow u$ in $W^{1, p}$ weakly. Let $\sigma$ be a weak limit of $\left|\nabla u_{n}\right|^{p-2} \nabla u_{n}$ in $L^{p^{\prime}}$. It satisfies

$$
-\operatorname{div} \sigma+k|u|^{p-2} u=H(x, u) .
$$

Multiplying this by $u$ and integrating by parts one gets

$$
\int_{\Omega} \nabla u . \sigma+k \int_{\Omega}|u|^{p}=\int_{\Omega} H(x, u) u .
$$

and on another hand passing to the limit in the equation satisfied by $u_{n}$, multiplied by $u_{n}$, one has

$$
\lim \int_{\Omega}\left|\nabla u_{n}\right|^{p}+k \int_{\Omega}|u|^{p}=\int_{\Omega} H(x, u) u .
$$

We have obtained that

$$
\int_{\Omega} \sigma \cdot \nabla u=\lim \int_{\Omega}\left|\nabla u_{n}\right|^{p}
$$

By using lower semicontinuity for the weak topology,

$$
\left|\int_{\Omega} \sigma \cdot \nabla u\right| \leq \lim \left(\int_{\Omega}\left|\nabla u_{n}\right|^{p}\right)^{\frac{p}{p-1}}\left(\int|\nabla u|^{p}\right)^{\frac{1}{p}}
$$

and then

$$
\lim \int_{\Omega}\left|\nabla u_{n}\right|^{p} \leq \lim \left(\int_{\Omega}\left|\nabla u_{n}\right|^{p}\right)^{\frac{p}{p-1}}\left(\int|\nabla u|^{p}\right)^{\frac{1}{p}}
$$

hence

$$
\lim \left(\int_{\Omega}\left|\nabla u_{n}\right|^{p}\right)^{\frac{1}{p}} \leq\left(\int_{\Omega}|\nabla u|^{p}\right)^{\frac{1}{p}}
$$

Since the other inequality is always true, one obtains that the convergence is strong, $\sigma=|\nabla u|^{p-2} \nabla u$, and $u$ is a solution.

Acknowledgement: Part of this work was done while the second author was visiting the Mathematical Department of the University "La Sapienza", she would like to thank the laboratory for the invitation. 


\section{References}

[1] S. Alama and G. Tarantello, On semilinear elliptic equations with indefinite nonlinearities, Calc. Var. and Partial Differential Equations, 1 (1993), 439-475.

[2] W. Allegretto, Y.X. Huang, Principal eigenvalues and Sturm comparison via Picone's identity. J. Differential Equations 156 (1999), no. $2,427-438$.

[3] A. Anane, Simplicity and isolation of the first eigenvalue of the $p$ Laplacian with weight C. R. Acad. Sci. Paris Sér. I Math. 305 (1987), no. $16,725-728$.

[4] G. Arioli, F. Gazzola Some results on p-Laplacian equations with a critical growth term, Differential and Integral Equations, 11, (1998), 311-326.

[5] H.Berestycki, I. Capuzzo Dolcetta, L. Nirenberg Problémes Elliptiques indéfinis et Théorème de Liouville non-linéaires, C. R. Acad. Sci. Paris Sér. I Math. 317, 945-950, (1993).

[6] I. Birindelli, F. Demengel On some partial differential equation for non coercive functional and critical Sobolev exponent, Differential Integral Equations 15 (2002) 823-837.

[7] W. Chen, C.Li A priori estimates for prescribing scalar curvature equations, Ann. of Math. 48 (1997), 47-92.

[8] F. Demengel, E. Hebey On some nonlinear equation involving the p-Laplacian and some critical Sobolev exponent, Adv. Differential Equations 3 (1998), no. 4, 533-574.

[9] Di Benedetto $\mathcal{C}^{1+\alpha}$ regularity of weak solutions of degenerate elliptic equations, Non Linear Anal. Vol.7, 8, pp 827-850, 1983.

[10] P. Drabek, Y. X. Huang Multiplicity of positive solutions for some quasi linear elliptic equations in $\mathbb{R}^{N}$ with critical Sobolev exponent J. Differential Equations, 140 (1997) 106-132. 
[11] P. Drabek, S. Pohozaev Positive solutions for the pLaplacian : Application of the fibrering method, Proc. Roy. Soc. Edinburgh, 127 A, 703-726, (1997).

[12] J. Garcia Azorero, I. Peral Alonso Some results about the existence of a second Positive solution in a quasilinar critical problem , Indiana University Math. Journal; Vol.43, 3, (1994) p. 941-957.

[13] M. Guedda, L. Veron, Local and Global properties of solutions of quasilinear elliptic equations, J. Differential Equations, 76 (1988), 159189.

[14] M. Guedda, L. Veron, Quasilinear equations involving critical Sobolev exponents, Nonlinear Anal. 13 (1989), 879-902.

[15] E. Hebey Introduction à l'Analyse Non Linéaire sur les variétés Diderot Editeurs, Arts et Sciences, Paris 1997.

[16] E. Hebey, M. Vaugon Existence and multiplicity of nodal solutions for nonlinear elliptic equations with critical Sobolev growth. J. Funct. Anal. 119 (1994), no. 2, 298-318.

[17] J.L. Lewis Regularity of the derivatives of solutions to certain degenerate elliptic equations, Indiana University Math. Journal, 32 (1983), 849-858.

[18] P.L. Lions La méthode de compacité concentration, I et II , Revista Ibero Americana, vol. 1, n1, (1985) p; 145

[19] P. Lindqvist, A note on the nonlinear Rayleigh quotient, Analysis, algebra, and computers in mathematical research (Luleå, 1992), 223231, Lecture Notes in Pure and Appl. Math., 156, Dekker, New York, 1994.

[20] T. Ouyang On the positive solutions of semilinear equations of $\Delta u+$ $\lambda u+h u^{p}=0$ on compacts manifolds, part II, Indiana University Math. Journal, 40, 1083-1140, (1991).

[21] S. Pohozaev, L. Veron, Multiple positive solutions of some quasilinear Neumann problems, Appl. Anal. 74, (2000), 363-391. 
[22] M. Struwe Variational Methods. Springer Verlag (1990)

[23] P. Tolksdorff Regularity for a more general class of quasilinear elliptic equations, J. Differential Equations, 51 (1984), 126-150.

[24] Vasquez A strong maximum principle for some quasilinear elliptic equations, Appl. Math. Optim. 12: 191-202, (1984).

Isabeau Birindelli

Università di Roma "La Sapienza"

Piazzale Aldo moro, 5

00185 Roma, Italy

e mail: isabeau@mat.uniroma1.it

Françoise Demengel

Université de Cergy Pontoise,

Site de Saint-Martin, 2 Avenue Adolphe Chauvin

95302 Cergy Pontoise

e mail: Francoise.Demengel@math.u-cergy.fr 